\title{
PERFORMANCE CHARACTERISTIC MEMS-BASED IMUs FOR UAVs NAVIGATION
}

\author{
H. A. Mohamed ${ }^{\text {a }}$, J. M. Hansen ${ }^{\text {b }}$, M. M. Elhabiby ${ }^{c}$, N. El-Sheimy ${ }^{\text {a }}$, Abu B. Sesay ${ }^{\text {a, }}{ }^{*}$ \\ a Department of Geomatics Engineering, University of Calgary, Calgary, Alberta, Canada - (haytham.abdalla, elsheimy, \\ sesay)@ucalgary.ca \\ ${ }^{\mathrm{b}}$ Department of Engineering Cybernetics, Norwegian University of Science and Technology, 741 Trondheim, Norway - \\ jakob.mahler.hansen@itk.ntnu.no \\ ${ }^{c}$ Public Works Department, Ain Shams University, Cairo, Egypt - mmelhabi@ucalgary.ca
}

Commission I, ICWG I/Vb - UAV-g 2015

KEY WORDS: UAV, Navigation, Sensor fusion, INS/GPS, IMU, GPS, Magnetometer, Extended Kalman Filter, RTK.

\begin{abstract}
:
Accurate 3D reconstruction has become essential for non-traditional mapping applications such as urban planning, mining industry, environmental monitoring, navigation, surveillance, pipeline inspection, infrastructure monitoring, landslide hazard analysis, indoor localization, and military simulation. The needs of these applications cannot be satisfied by traditional mapping, which is based on dedicated data acquisition systems designed for mapping purposes. Recent advances in hardware and software development have made it possible to conduct accurate 3D mapping without using costly and high-end data acquisition systems. Low-cost digital cameras, laser scanners, and navigation systems can provide accurate mapping if they are properly integrated at the hardware and software levels. Unmanned Aerial Vehicles (UAVs) are emerging as a mobile mapping platform that can provide additional economical and practical advantages. However, such economical and practical requirements need navigation systems that can provide uninterrupted navigation solution. Hence, testing the performance characteristics of Micro-Electro-Mechanical Systems (MEMS) or low cost navigation sensors for various UAV applications is important research. This work focuses on studying the performance characteristics under different manoeuvres using inertial measurements integrated with single point positioning, RealTime-Kinematic (RTK), and additional navigational aiding sensors. Furthermore, the performance of the inertial sensors is tested during Global Positioning System (GPS) signal outage.
\end{abstract}

\section{INTRODUCTION}

Unmanned Aerial Vehicle (UAV) can be used as autonomously controlled aerial vehicle without human intervention with different airframe types and sizes. It can also be controlled remotely from Ground Control Station (GCS) or Radio Control (RC). UAVs are attractive for many researchers as an aerial platform can participate in data acquisition (Francesco, 2014). This is due to their ability and capability in various applications such as 3D mapping, reconnaissance, surveillance, rescue operations, urban planning, mining industry, infrastructure monitoring, landslide hazard analysis and pipelines inspection. Moreover, due to the significant difference in cost between the $\mathrm{UAV}$ and the traditional manned aerial vehicle, this introduced more domination for the UAV in many applications (Colomina, 2008). Thus, automation of the UAV is an important project nowadays in order to improve and increase its performance. The UAV autonomous system consists of guidance, navigation and control (GNC) units (Kim, 2006). The guidance and control algorithms depend on the navigation information because they build their decisions according to the navigation solution. Therefore, improving UAV's navigation solution is considered to be a crucial task. Navigation is the science of determining, at a given time, the vehicle's location and attitude. Consequently, updating the Inertial Measurement Unit (IMU), as a main navigation sensor, with navigation aiding sensors such as Global Positioning System (GPS), magnetometer, or pressure sensor increases the UAV's navigation accuracy because the sensor fusion continually correct the navigation error drift caused by using the IMU sensor alone.

Moreover, if the UAV is used in a photogrammetry application the accuracy of the photogrammetry will increase with a reliable and accurate navigation solution (Sebastian, 2014). Thence, improving the UAV navigation will directly enhance the geomatics applications results.

Therefore, for good navigation assessment, this work focuses on studying and testing the performance characteristics of MEMS based or low cost navigation sensors under different manoeuvres using inertial measurements integrated with single point positioning, Real-Time-Kinematic (RTK) positioning, and additional navigational aiding (navaid) sensors. Furthermore, the performance of the inertial sensors is tested during GPS signal outage.

This paper is organized as follows: Section 2 describes system overview of the UAV and RTK positioning. Section 3 illustrates the used methodology. The comparisons of the experimental results are demonstrated in Section 4, and finally the conclusion is given in Section 5.

\section{SYSTEM OVERVIEW}

Experimental data was acquired using a fixed-wing UAV with sensor payload at Eggemoen Airport, Norway.

\subsection{UAV and Payload}

The fixed-wing UAV is a Penguin B manufactured by UAV Factory, with a payload of up to $10 \mathrm{~kg}$, flight time 20 hours and a maximum speed of $36 \mathrm{~m} / \mathrm{s}$. The payload consists of two IMUs and a Global Navigation Satellite System (GNSS) receiver accurately synchronised with a microcontroller. The GNSS receiver used is a u-Blox EVK-6T supplying estimated position as well as raw measurements, such as carrier-phase and pseudo-

\footnotetext{
* Corresponding author
} 
ranges. The IMUs (ADIS 16488 and STIM 300) are MEMSbased inertial sensors of tactical grade and supply specific force and angular rate, where the ADIS 16488 additionally has a built-in magnetometer. The IMU specifications can be found in the appendix.

\subsection{Real-Time-Kinematic Positioning}

An additional GNSS-receiver identical to the on-board receiver was placed at the base station throughout the experiments, making RTK positioning available. Evaluating the RTK position was done using the open-source program: RTKLIB (Takasu, 2007).

RTKLIB supplies the estimated position and divides it into three categories depending on position accuracy; single (single receiver accuracy), float (using differential positioning without a solution to the ambiguities) and fixed (using differential positioning with solved ambiguities). The UAV position is shown in Figure 1, where the position prior to take-off (approximately at 750 seconds) and after landing (approximately 2000 seconds) has single resolution, whereas fixed resolution is achieved throughout most of the flight.
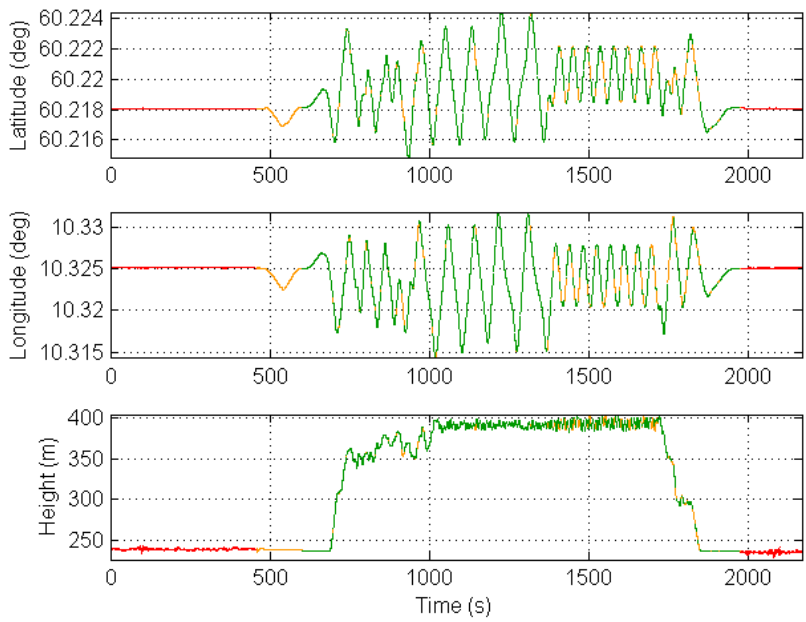

Figure 1. RTK position of UAV (Single: Red, Float: Yellow, Fixed: Green)

The flight includes several figures-of-eight and circles (14001700 seconds) performed by the autopilot. The take-off and landing were performed manually whereas the rest of the flight was controlled by the autopilot.

\section{METHODOLOGY}

\subsection{Wavelet De-noising}

The work is initiated by wavelet de-noising ADIS and STIM IMUs raw measurements in order to decrease the noise level by separation of the high and low frequency of the inertial sensor noise components (Sameh, 2003). This improves the navigation solution as described in the experimental results, Section 4.

Figure 2 and Figure 3 depict the gyro and accelerometer raw measurements for the ADIS IMU before and after de-noising. The IMU raw measurements before de-noising are represented by the blue plot, while the red plot presents the raw measurements after de-noising.
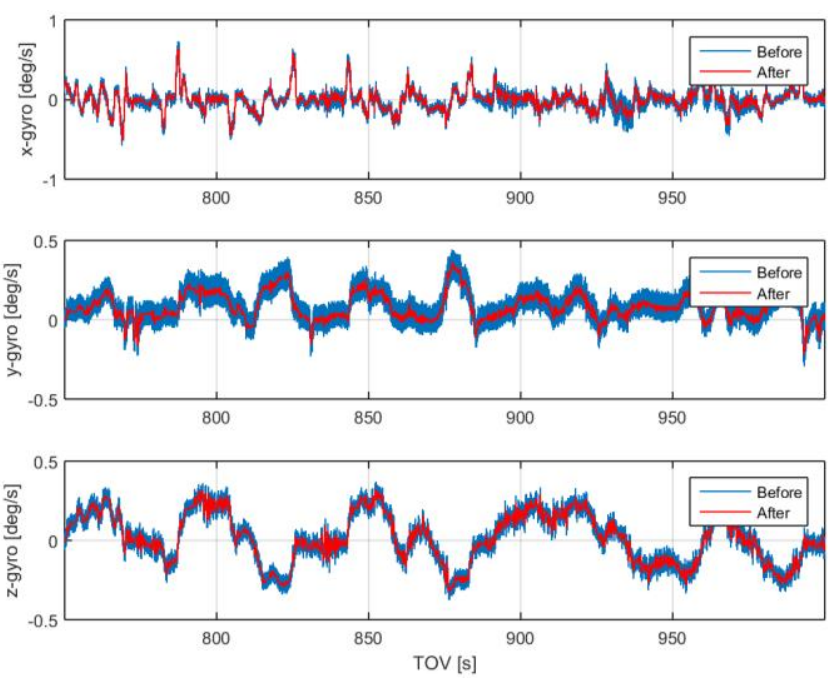

Figure 2. ADIS gyro measurements before and after de-noising
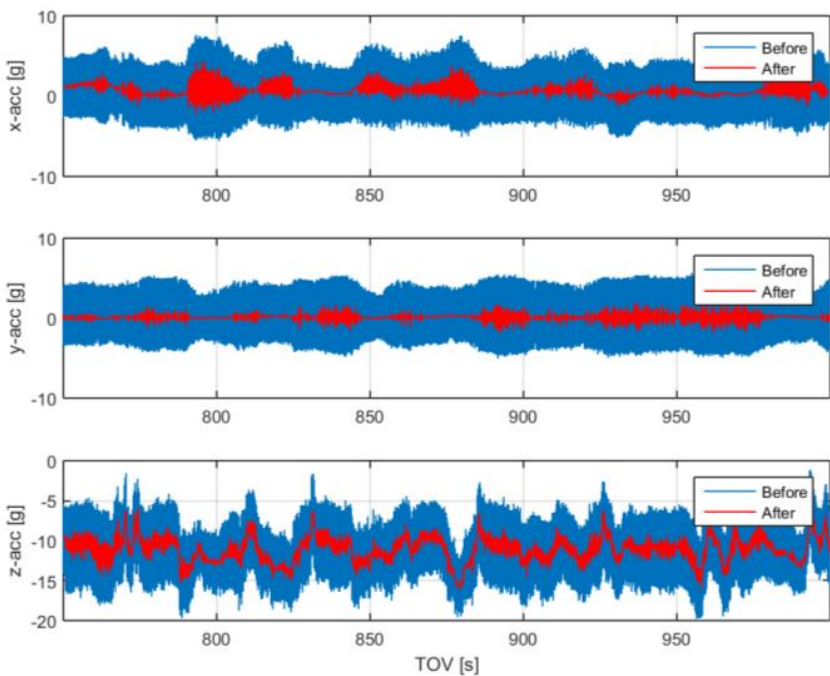

Figure 3. ADIS accelerometer measurements before and after de-noising

\subsection{Navigation Sensor Fusion}

The inertial navigation system is a standalone system. Although, it is a time dependent system, in which the error in an inertial sensor accumulates with time; it has good short time accuracy. Therefore, updating the system with navigation aided information such as GPS will improve the system accuracy during long time periods.

There are different forms of INS/GPS integration for instance loosely coupled, tightly coupled, and ultra-tightly coupled. This work used the loosely coupled architecture; it is also called decentralized integration (Noureldin, 2012). The INS and GPS work independently and provide its solutions. The INS provides the main navigation information such as position, velocity, and attitude whilst the GPS provides aiding information during availability such as absolute position and, velocity. A common method for integrating the sensor information from INS and GPS is the Kalman Filter (KF) or the Extended Kalman Filter $(E K F)$, which will give an improved navigational solution, compared to using either sensor information alone. 
Figure 4 illustrates closed loop loosely coupled INS/GPS integration. The corrected navigation solutions are fed back to the INS in order to correct the INS in each loop. Moreover, the position, velocity, and attitude of the EKF estimates are reset to zero after feeding back the error estimates.

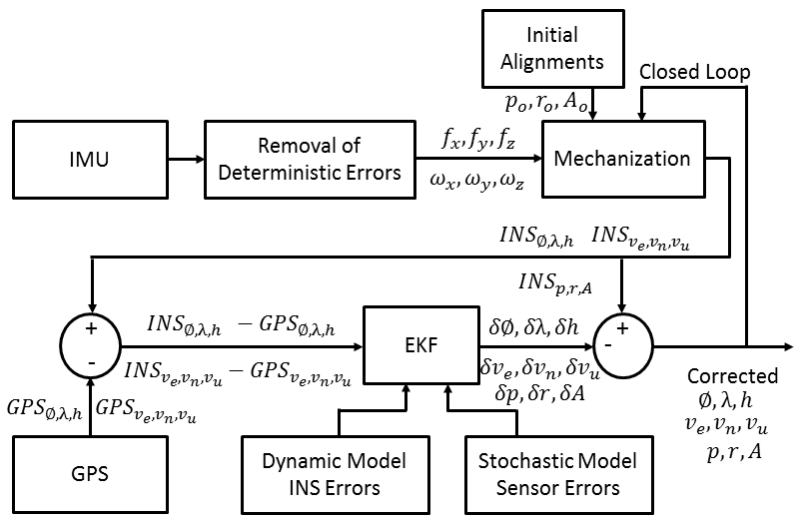

Figure 4. Closed loop loosely coupled integration

This work used two MEMS sensors (i.e., ADIS and STIM) (AnalogDevices) and (Sensonor). Typically, they have large drift in the navigation solution as shown in the results. Thus, the integration is achieved in different ways using EKF because the feedback loop is an important configuration for MEMS based INS operation (Yang, 2006). This is accomplished using the Aided Inertial Navigation System (AINS ${ }^{\mathrm{TM}}$ ) toolbox (Shin, 2004). The toolbox is developed at the Mobile Multi-Sensor Systems (MMSS) group at the University of Calgary.

The Kalman Filter describes the different INS error states by using the following first order state equation:

$$
\dot{x}=F x+G w
$$

where $\mathrm{x}=$ state estimate vector

$$
\begin{aligned}
& \mathrm{F}=\text { dynamic matrix } \\
& \mathrm{G}=\text { noise distribution matrix } \\
& \mathrm{W}=\text { process noise vector }
\end{aligned}
$$

The two terms on the right hand side respectively define the dynamic model and the stochastic model. The dynamic model describes how the states develop through time. On the other hand, the stochastic model defines the uncertainty in the dynamics model. Commonly, the INS/GPS integration using EKF has fifteen or twenty one states in order to determine 3D navigation solution (Shin, 2005). In this work, twenty one states are used as presented in equation (1) as each error is represented in $3 \mathrm{D}$ with respect to the navigation frame:

$$
x=\left[\begin{array}{lll}
\delta r \delta v & \delta \varepsilon \omega_{b} \delta f_{b} \delta \omega_{s} \delta f_{s}
\end{array}\right]
$$

where $\delta \mathrm{r}=$ position errors

$\delta \mathrm{v}=$ velocity errors

$\delta \varepsilon=$ attitude errors

$\delta \omega_{\mathrm{b}}=$ gyro bias errors

$\delta \mathrm{f}_{\mathrm{b}}=$ accelerometer bias errors

$\delta \omega_{\mathrm{s}}=$ gyro scale factor errors

$\delta \mathrm{f}_{\mathrm{s}}=$ accelerometer scale factor errors

Therefore, the corresponding discrete-time linear system can be expressed as follows:

$$
x_{k}=\Phi_{k, k-1} x_{k-1}+G_{k-1} w_{k-1}
$$

where $\mathrm{x}_{\mathrm{k}}=$ state estimate vector

$\Phi_{\mathrm{k}, \mathrm{k}-1}=$ transition matrix

$\mathrm{G}_{\mathrm{k}-1}=$ noise distribution matrix

$\mathrm{w}=$ process noise vector

$\mathrm{k}=$ current epoch

For INS compensation, GPS as an external observation of better accuracy are utilized. Equation (4) presents this observation:

$$
z_{k}=H_{k} x_{k}+\delta n_{k}
$$

where $\mathrm{z}_{\mathrm{k}}=$ observation vector

$\mathrm{H}_{\mathrm{k}}=$ design matrix

$\mathrm{x}_{\mathrm{k}}=$ state estimate vector

$\delta n_{\mathrm{k}}=$ observation noise

\subsection{Smoothing}

In this section, the EKF data results are post-processed using the Rauch-Tung-Striebel smoother (RTS) algorithm (Robert, 1996), which is considered to be a fixed interval backwards smoother. This means that the entire interval of measurements is fixed and all measurements are available. Thus, the RTS algorithm consists of a forward sweep; this is done by EKF, then followed by a backwards sweep which begins at the end of the forward filter with the initial conditions of the last epoch, as shown in Figure 5. The aim of RTS smoother is to enhance the accuracy.

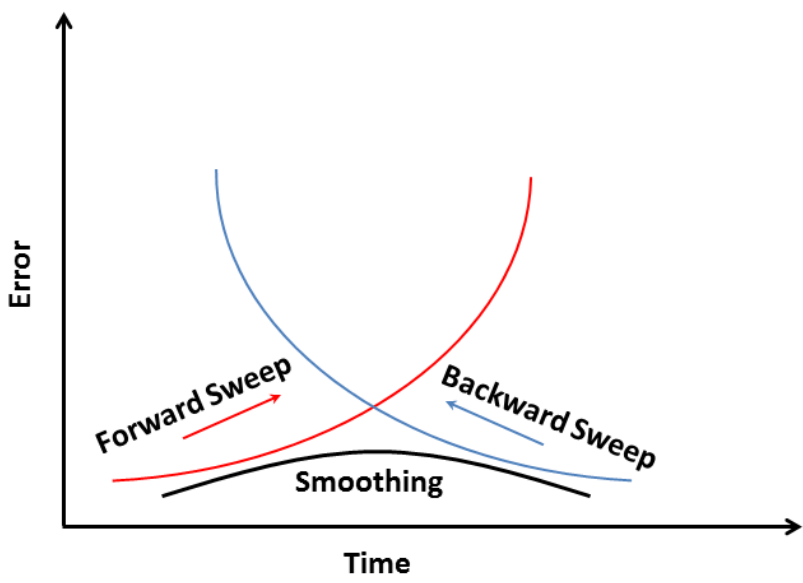

Figure 5. The effect of the RTS smoothing

\section{EXPERIMENTAL RESULTS}

As mentioned previously, the integration is achieved in different ways using EKF:

- First, the INS/RTK integration with the aid of the magnetometer is carried out for both IMUs. The RTK in this case provides positon and velocity update. The smoothing result by using Rauch-Tung-Striebel smoother (RTS) algorithm from the better IMU is selected to be the reference; it is denoted by (REF).

- $\quad$ Second, INS/RTK integration is performed for both IMUs but without the magnetometer as a navaid. 
- Third, INS/RTK integration is implemented for both IMUs. However, the RTK in this case updates the INS with position only.

- $\quad$ Fourth, INS/GPS integration is implemented for both IMUs. GPS supplies positon as an update to the INS (i.e., coordinate update "CUPT").

- $\quad$ Finally, INS/RTK and INS/GPS integrations are also performed during GPS signal outage for $30 \mathrm{sec}$ in order to study the performance of the inertial sensors.

These scenarios will be tested in the following sections.

\subsection{INS/GPS Integration without GPS Signal Outage}

Figure 6 illustrates the UAV trajectory results for approximately $35 \mathrm{~min}$ experimental flight between the reference and INS/RTK integration for both ADIS IMU and STIM IMU with position and velocity update. The Root Mean Square Error (RMSE) for the total trajectory of both ADIS and STIM IMUs are $0.07 \mathrm{~m}$ and $0.14 \mathrm{~m}$ respectively. The blue line represents the reference (REF), the red line presents the INS/RTK integration using the ADIS sensor, and finally the green line shows the INS/RTK integration using the STIM sensor.

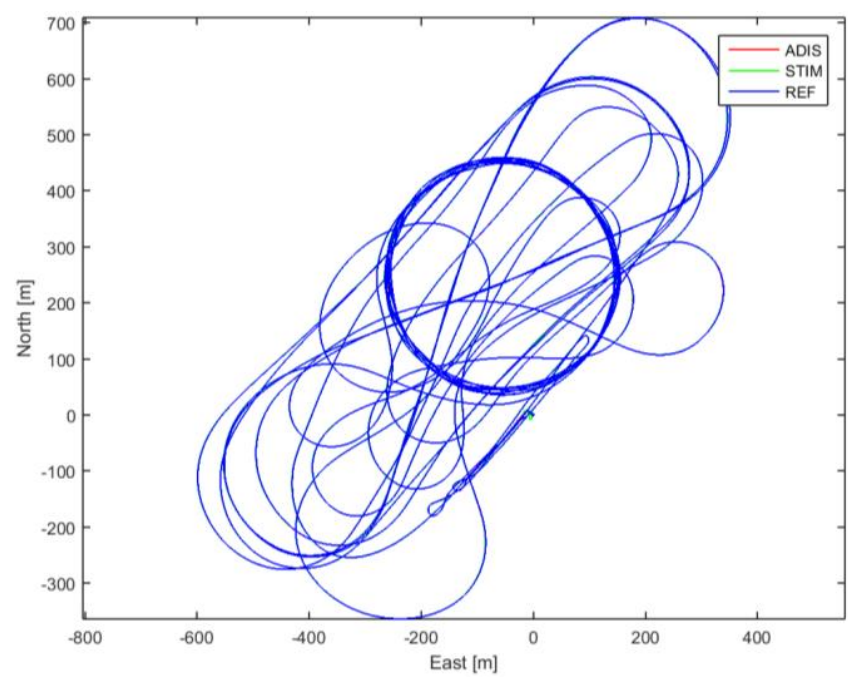

Figure 6. UAV trajectory results by using INS/RTK for both sensors ADIS, STIM and the reference

Figure 7 and Figure 8 demonstrate position and attitude error between the reference and INS/RTK integration for both ADIS and STIM IMUs with position and velocity update respectively. The ADIS sensor is represented by the red line, whilst the green line presents the STIM sensor. The position and attitude (PA) RMSEs for both sensors ADIS and STIM are explained in Table 1. The RMSEs are computed during the flight period which is after the take-off and before the landing.

\begin{tabular}{|c|c|c|c|c|c|c|}
\cline { 2 - 7 } \multicolumn{1}{c|}{} & \multicolumn{3}{c|}{ Position RMSE [m] } & \multicolumn{3}{c|}{ Attitude RMSE [deg] } \\
\cline { 2 - 7 } \multicolumn{1}{c|}{} & East & North & Down & Roll & Pitch & Azimuth \\
\hline ADIS & 0.02 & 0.03 & 0.05 & 0.09 & 0.09 & 0.15 \\
\hline STIM & 0.06 & 0.07 & 0.10 & 0.36 & 0.68 & 0.58 \\
\hline
\end{tabular}

Table 1. PA RMSE for both IMUs using RTK position and velocity update
The position and attitude RMSE values in Table 1 show that the ADIS sensor gives better results than the STIM sensor in both position and attitude errors.
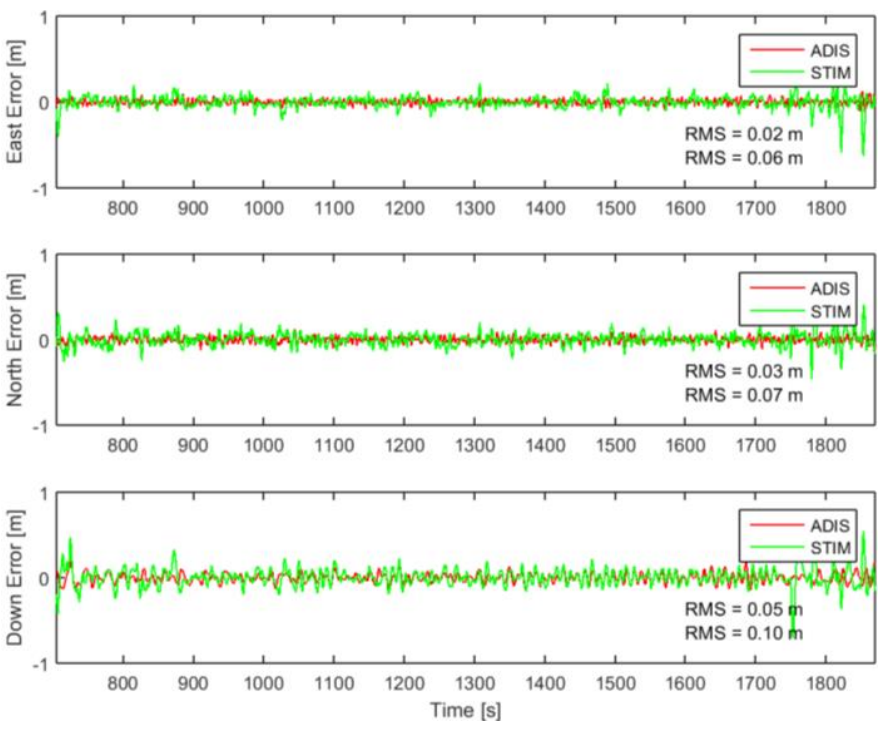

Figure 7. Position error in East, North, and Down for both IMUs
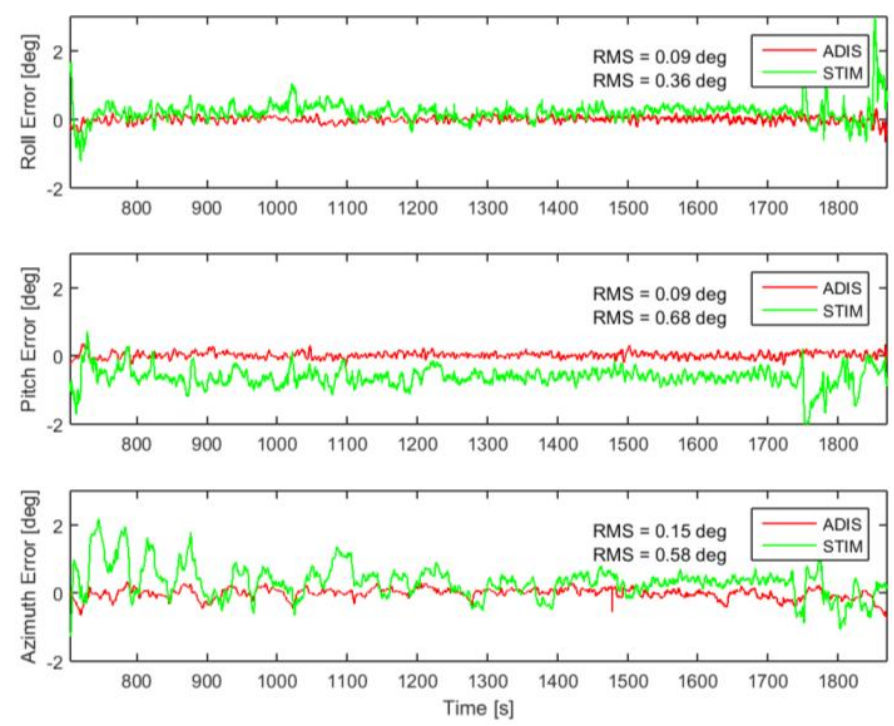

Figure 8. Roll, Pitch, and Azimuth errors for ADIS and STIM sensors

Figure 9 represents the azimuth convergence time using both ADIS and STIM IMUs, they are approximately the same. Thus, the UAV have to loiter or remain on the tarmac for approximately $7 \mathrm{~min}$ after alignment and before initializing the mission. The period in between the blue lines presents the flight period which is after the take-off and before the landing. The red line represents the ADIS IMU heading standard deviation, while the STIM IMU heading standard deviation is represented by the green line.

Table 2 illustrates the position and attitude RMSEs for different integration results for INS/RTK with position update and INS/GPS with position update as well. The integration is accomplished for both IMUs sensor. 


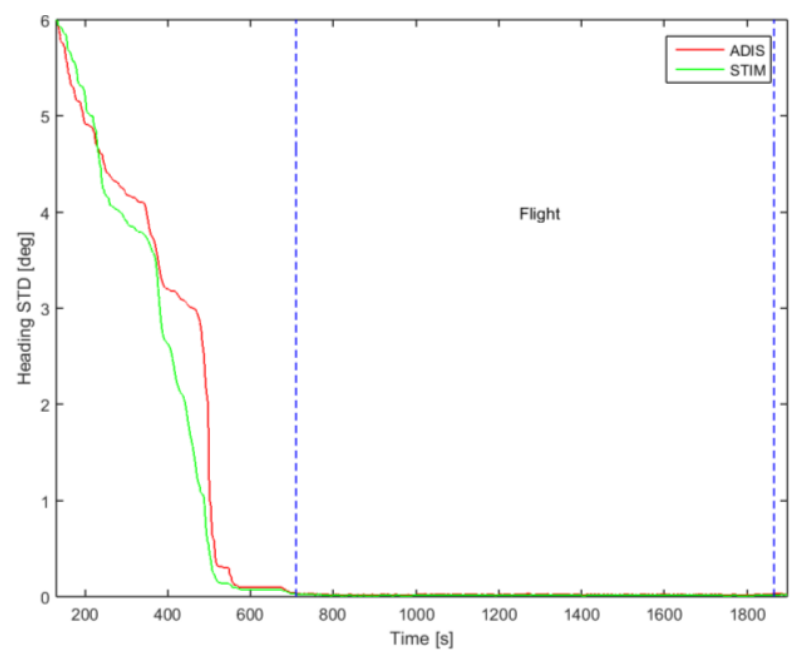

Figure 9. Azimuth convergence time for both IMUs

\begin{tabular}{|c|c|c|c|c|c|c|c|}
\cline { 3 - 8 } \multicolumn{2}{c|}{} & \multicolumn{3}{|c|}{ Position RMSE [m] } & \multicolumn{3}{|c|}{ Attitude RMSE [deg] } \\
\cline { 3 - 8 } \multicolumn{2}{c|}{} & East & North & Down & Roll & Pitch & Azimuth \\
\hline \multirow{3}{*}{ ADIS } & RTK & 0.02 & 0.03 & 0.05 & 0.09 & 0.09 & 0.15 \\
\cline { 2 - 8 } & GPS & 8.02 & 8.98 & 1.42 & 0.64 & 0.79 & 2.56 \\
\hline \multirow{3}{*}{ STIM } & RTK & 0.06 & 0.07 & 0.10 & 0.37 & 0.69 & 0.51 \\
\cline { 2 - 8 } & GPS & 8.28 & 9.45 & 1.48 & 0.67 & 0.79 & 2.24 \\
\hline
\end{tabular}

Table 2. PA RMSE for the IMUs using RTK or GPS position update

From Table 2 it is clear that the two IMUs contribute similarly to the system accuracy when GPS is used. However, the ADIS IMU performs better than the STIM IMU when RTK positioning is used for aiding. In general both using RTK instead of single point positioning reduces the RMSE.

Table 3 demonstrates the position and attitude RMSEs for the ADIS IMU before and after de-noising in order to show the impact of the wavelet de-noising.

\begin{tabular}{|c|c|c|c|c|c|c|}
\cline { 2 - 7 } \multicolumn{1}{c|}{} & \multicolumn{3}{c|}{ Position RMSE [m] } & \multicolumn{3}{c|}{ Attitude RMSE [deg] } \\
\cline { 2 - 7 } \multicolumn{1}{c|}{} & East & North & Down & Roll & Pitch & Azimuth \\
\hline Before & 0.35 & 0.35 & 0.10 & 0.13 & 0.13 & 0.24 \\
\hline After & 0.02 & 0.03 & 0.05 & 0.09 & 0.09 & 0.15 \\
\hline
\end{tabular}

Table 3. PA RMSE for ADIS before and after de-noising

The RMSE values for using de-noised signals are lower than the noisy measurements, as seen in Table 3 . This is expected.

\subsection{INS/GPS Integration with GPS Signal Outage}

Figure 10 shows the performance of the INS/RTK for ADIS sensor with and without magnetometer during RTK signal outage of $30 \mathrm{sec}$. The RTK updates the INS with position and velocity in both scenarios. The position RMS errors between the INS/RTK integration with magnetometer and without magnetometer with the reference during the RTK signal outage period are $11.5 \mathrm{~m}$ and $15.0 \mathrm{~m}$ respectively. The blue line stands for the reference, the green line shows the INS/RTK integration without magnetometer, and the INS/RTK integration with magnetometer is represented by red line.

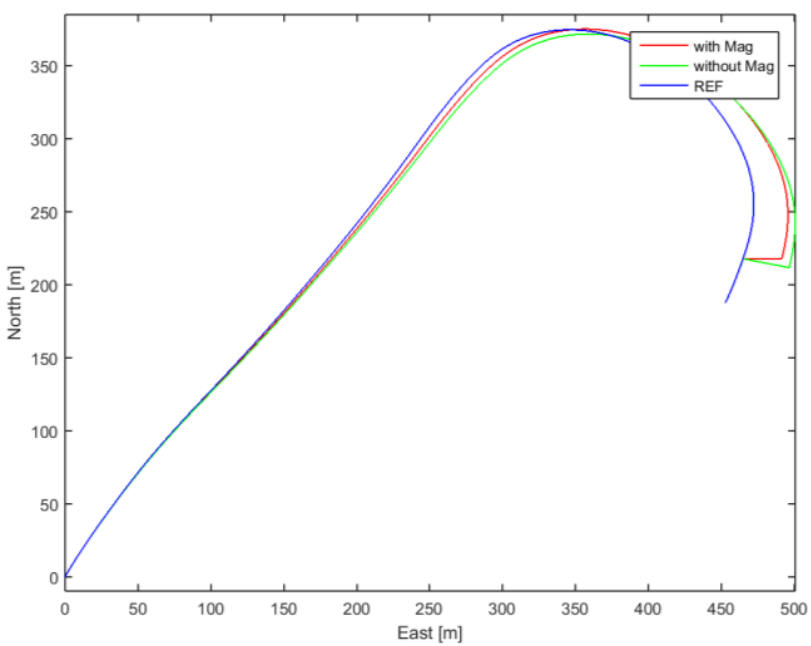

Figure 10. Portion of the trajectory during RTK signal outage using ADIS

Figure 11 depicts the position error in the East, North, and Down directions between the reference and the INS/RTK integration with magnetometer and without magnetometer during the RTK signal outage period. The RMS errors in the East, North, and Down directions are 11.53, 0.50, $0.81 \mathrm{~m}$ and 14.51, 3.18, $2.07 \mathrm{~m}$ respectively as shown in Table 4. The green line states the INS/RTK integration without magnetometer, and the INS/RTK integration with magnetometer is presented by the red line.
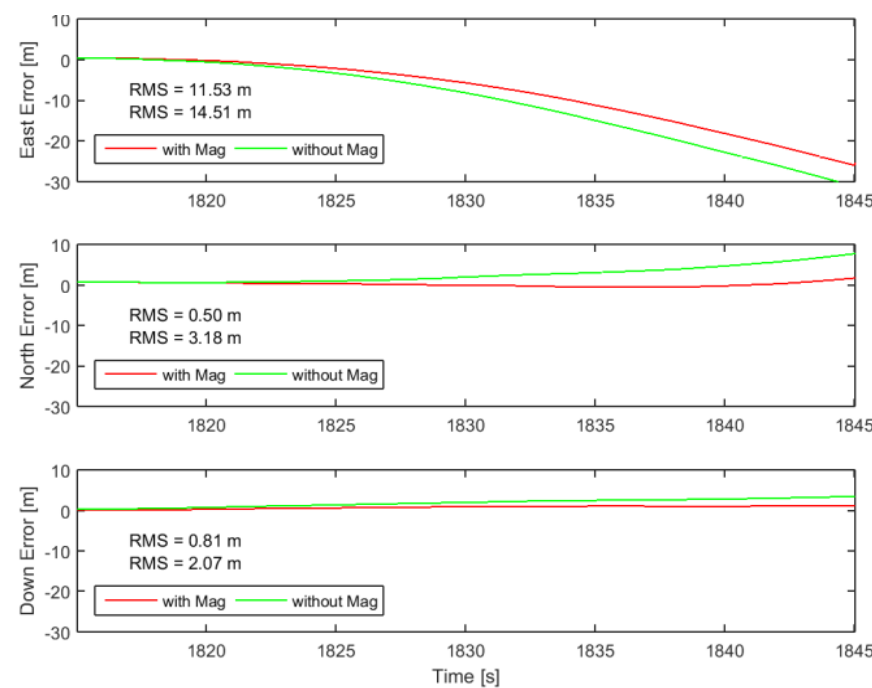

Figure 11. Position error in East, North, and Down during RTK signal outage

Figure 12 demonstrates the attitude error between the reference and the INS/RTK integration with magnetometer and without magnetometer during the RTK signal outage period. The roll, pitch, and azimuth RMS errors are $0.19,0.23,0.14 \mathrm{~m}$ and 0.28 , $0.26,0.14$ degree respectively as shown in Table 4 . The green line represents the INS/RTK integration without magnetometer, and the INS/RTK integration with magnetometer is presented by the red line. 

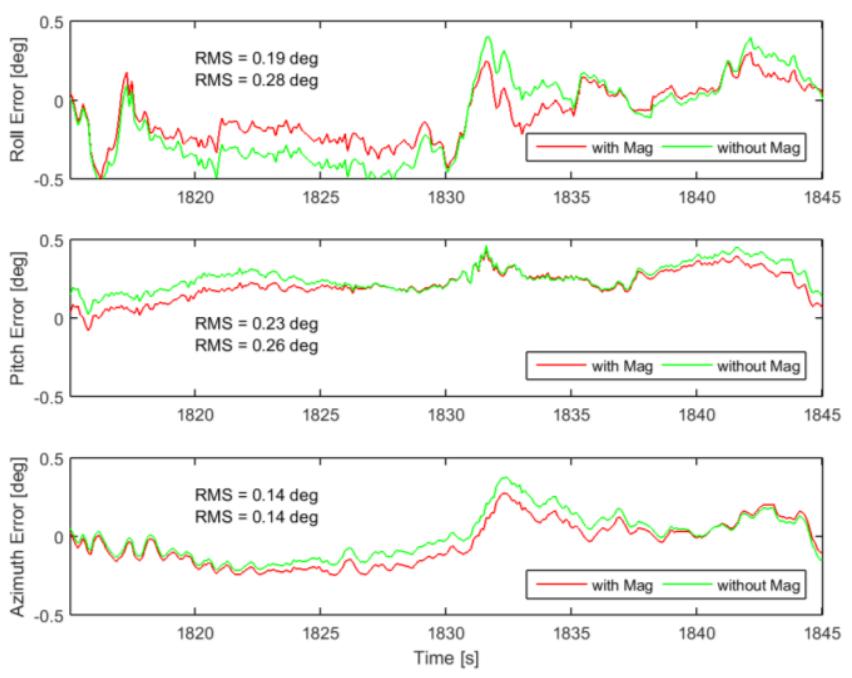

Figure 12. Attitude errors for ADIS IMU during RTK signal outage

\begin{tabular}{|c|c|c|c|c|c|c|}
\cline { 2 - 7 } \multicolumn{1}{c|}{} & \multicolumn{3}{c|}{ Position RMSE [m] } & \multicolumn{3}{c|}{ Attitude RMSE [deg] } \\
\cline { 2 - 7 } \multicolumn{1}{c|}{} & East & North & Down & Roll & Pitch & Azimuth \\
\hline $\begin{array}{c}\text { With } \\
\text { Mag. }\end{array}$ & 11.53 & 0.50 & 0.81 & 0.19 & 0.23 & 0.14 \\
\hline $\begin{array}{c}\text { Without } \\
\text { Mag. }\end{array}$ & 14.51 & 3.18 & 2.07 & 0.28 & 0.26 & 0.14 \\
\hline
\end{tabular}

Table 4. ADIS PA RMSE for magnetometer aiding using RTK position and velocity update

Table 4 shows a clear advantage of using magnetometer during periods of missing satellite signals. The positioning is improved significantly; however the attitude is less affected, but still improved.

Table 5 illustrates position and attitude RMSEs during RTK/GPS signal outage for different scenarios. First, INS/RTK integration with/without magnetometer, RTK provides position update. Second, INS/GPS integration with/without magnetometer and GPS also supplies position update.

\begin{tabular}{|c|c|c|c|c|c|c|c|}
\hline & \multicolumn{3}{|c|}{ Position RMSE [m] } & \multicolumn{3}{|c|}{ Attitude RMSE [deg] } \\
\hline & & East & North & Down & Roll & Pitch & Azimuth \\
\hline \multirow{2}{*}{$\begin{array}{l}\text { INS/ } \\
\text { RTK }\end{array}$} & $\begin{array}{l}\text { With } \\
\text { Mag }\end{array}$ & 11.54 & 0.50 & 0.81 & 0.19 & 0.23 & 0.14 \\
\hline & $\begin{array}{c}\text { Without } \\
\text { Mag }\end{array}$ & 14.63 & 3.37 & 2.12 & 0.28 & 0.27 & 0.14 \\
\hline \multirow{2}{*}{$\begin{array}{l}\text { INS/ } \\
\text { GPS }\end{array}$} & $\begin{array}{l}\text { With } \\
\text { Mag } \\
\end{array}$ & 9.73 & 62.37 & 4.40 & 0.17 & 0.20 & 0.23 \\
\hline & $\begin{array}{c}\text { Without } \\
\text { Mag }\end{array}$ & 8.42 & 53.94 & 2.67 & 0.23 & 0.25 & 0.24 \\
\hline
\end{tabular}

Table 5. ADIS PA RMSE for magnetometer aiding, GPS and RTK position update during satellite signal outage

\section{CONCLUSION}

In this work the performance characteristics of MEMS based is studied under different manoeuvres using inertial measurements integrated with single point positioning, RTK, and magnetometer.
It was found that using RTK positioning in the integration procedure gives higher accuracy. Furthermore, it was seen that de-noising of the IMU signals might be advantageous in order to reduce the RMS error. For periods with outage of satellite signals, it was shown to be advantageous to have the magnetometer heading as an aiding sensor.

\section{ACKNOWLEDGEMENTS}

The data used in this paper is provided by the Norwegian Research Council (projects no. 221666 and 223254) through the Centre of Autonomous Marine Operations and Systems (AMOS) at the Norwegian University of Science and Technology.

\section{REFERENCES}

ADIS-16488A IMU sensor data sheet. Report on Analog Devices.

http://www.analog.com/media/en/technical-documentation/datasheets/ADIS16488A.pdf

Colomina I., Blázquez M., Molina P., Parés M. E., and Wis M., 2008. Towards a new paradigm for high-resolution low-cost photogrammetry and remote sensing. In: The International Archives of Photogrammetry, Remote Sensing and Spatial Information Sciences, Beijing, China, Vol. XXXVII, Part B1, pp. 1201-1206.

Francesco N., and Fabio R., 2014. UAV for 3D mapping applications: a review. Applied Geomatics, 6(1), pp. 1-15.

Kim J. H., Wishart S., and Sukkarieh S., 2006. Real-time Navigation, Guidance, and Control of a UAV using Low-cost Sensors. Field and Service Robotics Springer Tracts in Advanced Robotics, 24, pp. 299-309.

A. Noureldin, T. B. Karamat, and J. Georgy, 2012. Fundamentals of Inertial Navigation, Satellite-based Positioning and their Integration. Springer Heidelberg New York Dordrecht London, pp. 15-17.

Robert Grover Brown, and Patrick Y. C. Hwang, 1996. Introduction to Random Signals and Applied Kalman Filtering. $3^{\text {rd }}$ edition, John Wiley \& Sons, pp. 312-330.

Sameh Nassar, 2003. Improving the Inertial Navigation System (INS) Error Model for INS and INS/DGPS Applications. PhD Thesis, Department of Geomatics Engineering, University of Calgary, Calgary, AB, Canada, pp. 62-72.

Sebastian S., Jochen T., 2014. Mobile 3D mapping for surveying earthwork projects using an Unmanned Aerial Vehicle (UAV) system. Automation in Construction, 41, pp. 1-14.

STIM-300 IMU sensor data sheet. Report on Sensonor. http://www.sensonor.com/media/99614/ts1524.r19\%20datasheet $\% 20$ stim300.pdf

Shin E., N. El-sheimy, 2004. Report on the Innovate Calgary "Aided Inertial Navigation System (AINS ${ }^{\mathrm{TM}}$ ) Toolbox", Calgary, Canada http://www.innovatecalgary.com/files/file/524-7-aidedinertial-navigation-system-ains-toolbox.pdf. 
Shin E., X. Niu, and N. El-Sheimy, 2005. Performance Comparison of the Extended and the Unscented Kalman Filter for Integrated GPS and MEMS-Based Inertial Systems. In: ION NTM, San Diego, CA, USA, pp. 961-969.

Takasu T., Kubo N., and Yasuda A., 2007. Report on the GPS/GNSS Symposium "RTKLIB An Open Source Program Package for GNSS Positioning", Tokyo, Japan http://www.rtklib.com (20-22 Nov. 2007).

Yang Y., X. Niu, and N. El-Sheimy, 2006. Real-Time MEMS Based INS/GPS Integrated Navigation System for Land Vehicle Navigation Application. In: ION NTM, Monterey, California, USA, pp. 501-507.

\section{APPENDIX}

\section{Used IMUs Specifications:}

\section{ADIS-16488 IMU:}

In-run Gyro Rate Bias Stability Angular Random Walk

In-run Accelerometer Bias Stability Velocity Random Walk

\section{STIM-300 IMU:}

In-run Gyro Rate Bias Stability Angular Random Walk

In-run Accelerometer Bias Stability Velocity Random Walk

$$
\begin{aligned}
& 6 \mathrm{deg} / \mathrm{hr} \\
& 0.3 \mathrm{deg} / \sqrt{\mathrm{hr}} \\
& 0.1 \mathrm{mg} \\
& 0.029 \frac{\mathrm{m}}{\mathrm{s}} / \sqrt{\mathrm{hr}} \\
& 0.5 \mathrm{deg} / \mathrm{hr} \\
& 0.15 \mathrm{deg} / \sqrt{\mathrm{hr}} \\
& 0.05 \mathrm{mg} \\
& 0.06 \frac{\mathrm{m}}{\mathrm{s}} / \sqrt{\mathrm{hr}}
\end{aligned}
$$

